\title{
Transcriptome sequence and physiological analysis revealed the roles of carotenoids and photosynthesis under low temperature combined with low-light stress on pepper (Capsicum annuum L.)
}

\author{
J. LI, J.M. XIE ${ }^{+}$(D) , J.H. YU, J. LYV, E.P. BAKPA, X.D. ZHANG, J. ZHANG, C.N. TANG, D.X DING, \\ N.H. LI, F. GAO, and C. WANG \\ College of Horticulture, Gansu Agricultural University, Yingmen Village, Anning District, 730070 Lanzhou, China
}

\begin{abstract}
Low temperature combined with low light (LL) is an adverse factor seriously affecting pepper productivity and quality. However, little is known about the molecular mechanisms related to LL stress responses. Therefore, transcriptome and physiological changes in Hangjiao No. 2 (H2) and Xiangtela No. 2 (X2) pepper were studied under normal conditions and LL. We found 8,392 and 8,028 differentially expressed genes in H2 and X2, respectively, significantly associated with photosynthesis, photosynthesis antenna proteins, and carotenoids, were enriched in 27 and 40 gene ontology terms in $\mathrm{H} 2$ and X2, respectively, and 14 and 16 Kyoto Encyclopedia of Gene and Genomes pathways. The accuracy and reliability of the RNA-Seq results were confirmed by qRT-PCR. Furthermore, carotenoid-related genes ZDS, CA1, CA2, NCED, LOC107840293, and LOC107850059 functioned in response to LL. Additionally, LL significantly decreased photosynthesis capacity, photosynthetic pigment contents, as well as maximum quantum efficiency, and changed carotenoid-related compounds, revealing photosynthesis and carotenoids were involved in LL stress response. Our findings provide insight into LL stress-induced transcriptional expression patterns.
\end{abstract}

Keywords: carotenoid metabolism; pepper; photosynthesis; transcriptome; zeaxanthin.

\section{Introduction}

Plants are continuously exposed to various environmental stresses throughout their growth. Abiotic stresses, such as drought, heat, chilling, salinity, high light, low light, and nutrient stresses, affect plants growth and yield (Zhao et al. 2013, Campany et al. 2016, Pedersen et al. 2016, Tricker et al. 2016, Truffault et al. 2016, Abdelaal et al.

\section{Highlights}

- Photosynthesis and carotenoids were involved in LL stress response in pepper

- LL decreased photosynthesis capacity and changed carotenoid-related compositions

- Carotenoid-related genes ZDS, CA1, CA2, NCED, LOC107850059 functioned in response to $\mathrm{LL}$
Received 29 September 2020

Accepted 1 December 2020

Published online 18 December 2020

${ }^{+}$Corresponding author

e-mail: xiejianminggs@126.com phone: +8613893335780

Abbreviations: ABA - abscisic acid; Ant - antheraxanthin; BP - biological process; Car - carotenoid content; $\mathrm{CC}$ - cellular component; $\mathrm{Chl}$ - chlorophyll; $\mathrm{CHY} \beta$ - $\beta$-carotenoid hydroxylase; $C_{\mathrm{i}}$ - intercellular $\mathrm{CO}_{2}$ concentration; $\mathrm{CI}$ - chilling injury; DEG - differentially expressed gene; $E$ - transpiration rate; $\mathrm{F}_{0}$ - minimal chlorophyll fluorescence; $\mathrm{F}_{\mathrm{m}}$ - maximal chlorophyll fluorescence; FPKM fragments per kilobase of transcript sequence per millions base pairs sequenced; $F_{v}-$ variable chlorophyll fluorescence; $F_{v} / F_{m}-$ maximal photosystem quantum yield; GO - gene ontology; $g_{\mathrm{s}}$ - stomatal conductance; HPLC - high-performance liquid chromatography; KEGG - Kyoto Encyclopedia of Genes and Genomes; LL - low temperature combined with low light; Lut - lutein; Lut-epoxide lutein-epoxide; Lyc - lycopene; MF - molecular function; NCED - 9-cis-epoxycarotenoid dioxygenase; Neo - neoxanthin; Phy phytoene; $P_{\mathrm{N}}$ - net photosynthetic rate; Vio - violaxanthin; Zea - zeaxanthin; ZEP - zeaxanthin cyclooxygenase; $\alpha$-Car - $\alpha$-carotenoid; $\beta$-Car - $\beta$-carotenoid.

Acknowledgments: This research was financially funded by the Scientific Research Start-Up Funds for Openly-Recruited Doctors (GAUKYQD-2018-35), National Natural Science Foundation of China (32072657), National Key Research and Development Program of China (2016YFD0201005), and Special Fund for Sci. \& Tech. Innovation and Development Guided by Gansu Province (2018ZX-02). The writers acknowledge all the authors, the editors, and the reviewers for precious and valuable comments and suggestions, which have greatly improved the article.

Conflict of interest: The authors declare that they have no conflict of interest. 
2018, 2020a; Kirilovsky and Büchel 2019, Hafez et al. 2020, Zhuang et al. 2019). Temperature and light are often correlated(Legris etal.2017) and low temperature combined with low light (LL) is a widespread environmental stress factor that adversely affects plant growth, development, and distribution (Sanghera et al. 2011, Ou et al. 2015). Over the past five years, it has been estimated that more than 67,000 ha of all kind of facilities were added each year and there are 3.685 million ha vegetable facility areas now in China, while plants grown in greenhouses especially, suffer LL during fall-winter and winter-spring seasons in north China. This condition causes physiological disorders which significantly limit their market value, decrease crop productivity, and economic income (Li et al. 2016). As the number of vegetable facility areas increases, the problem of LL stress urgently needs to be addressed.

Carotenoids are a large group of color pigments ranging from yellow to red widely present in plants, fungi, and algae (Rodriguez-Amaya 2016), which are responsible for the color formation, photosynthesis, and phytohormones (strigolactone and abscisic acid) (Cazzonelli and Pogson 2010). Numerous studies on carotenoids have focused on food science, and most of them were attributed to the changes of concentrations in fruits or vegetables during the maturity and processing cycle, such as in tomato, orange, watermelon, apple, apricot, medlar, persimmon, red grape, yellow or red pepper, carrot, and pumpkin (Daood et al. 2014, Fratianni et al. 2015, Kim et al. 2016). Additionally, the changes in lutein (Lut), $\beta$-carotene ( $\beta$-car), violaxanthin (Vio), and neoxanthin (Neo) in leaf alter photosynthesis, antenna assembly, as well as photoprotection (DemmigAdams and Adams 2006, Dall'Osto et al. 2010). A study conducted by Kang et al. (2018) showed overexpression of lycopene $\beta$-cyclase (IbLCYB2) significantly improved the contents of $\alpha$-carotene ( $\alpha$-Car), $\beta$-Car, Lut, $\beta$-cryptoxanthin, and zeaxanthin (Zea) leading to enhancement of salt tolerance (Kang et al. 2018). Ke et al. (2019) also reported lycopene epsilon-cyclase (IbLCY-epsilon) gene downregulation in transgenic sweet potato can increase total carotenoid and $\beta$-Car contents, leading to the high activity of reactive oxygen species/radical scavenging, consequently, enhancing salt and drought tolerances (Ke et al. 2019). Whereas, little concern about the relationship between the carotenoids and LL resistance is reported.

Relevant process in various response mechanisms has been studied by physiological and molecular methods to estimate gene expression, physiological and biochemical changes in phytohormones, membrane lipid composition, and photosynthetic pigments (Thomashow 1999). Transcriptome sequencing is widely employed to detect and elucidate the molecular mechanisms involved in physiological and physiochemical processes, analyze genomes, and identify functional genes induced by stress (Wang et al. 2015). Tomato plants undergo an early response of transient changes in stress-related protein genes and subsequent stable changes in gene expression leading to photosynthesis adjustments under chilling stress (Barrero-Gil et al. 2016). An et al. (2012) identified 508 transcripts in apical shoots of cassava under $7^{\circ} \mathrm{C}$ for 0 , 4 , and $9 \mathrm{~h}$ for early cold-responsive genes, which were annotated into Gene Ontology (GO) categories of response to abiotic and biotic stimuli, transcription factor activity, and chloroplast, and 319 differentially expressed genes (DEGs) annotated to 44 Kyoto Encyclopedia of Genes and Genomes (KEGG) pathways of plant hormone signal, phenylalanine metabolism, photosynthesis, etc. (An et al. 2012).

Our previous studies found that LL causes changes in carotenoid-related compounds in response to LL (Zhang et al. 2020a), but the mechanism is unknown. Moreover, little information focused on transcriptome analysis of pepper (Capsicum annuum L.) in response to LL stress is reported till now. Therefore, in the present study, transcriptome and physiological analysis were conducted to reveal and identify functional genes of pepper seedlings leaves of two varieties with different LL tolerance levels. The findings aid our understanding of the molecular mechanisms of pepper seedlings to LL via studying pathways of photosynthesis and carotenoid-related metabolism.

\section{Materials and methods}

Plant materials and growth conditions: Two pepper cultivars, namely Hangjiao No. 2 (H2, LL-sensitive cultivar) and Xiangtela No. 2 (X2, LL-tolerant cultivar), were used. The two cultivars were selected from our previous study (unpublished) on changes in carotenoid composition in pepper leaves under low temperature and light in relationship with the tolerance of varieties by comparing 12 cultivars under $5 / 5^{\circ} \mathrm{C}$ for $20 \mathrm{~d}$. $\mathrm{H} 2$ was purchased from Chinese Academy of Agricultural Sciences and X2 was provided by Hunan Academy of Agricultural Science.

Pepper seeds with uniform size, plumpness, and no impurities were continuously stirred for $15 \mathrm{~min}$ in the water at $55^{\circ} \mathrm{C}$, then socked for $6 \mathrm{~h}$ at $25^{\circ} \mathrm{C}$, placed on a water-absorbent towel, then germinated on a moist towel under dark conditions at $28^{\circ} \mathrm{C}$. Two germinated seeds were sown in black plastic pots $(9 \times 9 \mathrm{~cm})$ filled with a seedling-raising substrate (vermiculite:grass carbon:cow dung $=3: 1: 1)$, grown at $28 / 18^{\circ} \mathrm{C}$ (day/night), a PPFD of $300 \mu \mathrm{mol} \mathrm{m} \mathrm{m}^{-2} \mathrm{~s}^{-1}$, and relative humidity of $65-70 \%$ in the greenhouse of Gansu Agricultural University (N3605'39.86", E103'42'31.09"), and kept on consistent water and fertilizer management.

Stress treatment: Uniform seedlings with seven fully expanded leaves (50 d after sowing) were moved into growth chambers $(R D N-400 E-4$, Zhejiang, China) for stress treatments. For control (CK), the temperature was $28 / 18^{\circ} \mathrm{C}$ (day/night), and the PPFD was $300 \mu \mathrm{mol} \mathrm{m}^{-2} \mathrm{~s}^{-1}$, while for low temperature combined with low-light (LL) treatment, the temperature was $15 / 5^{\circ} \mathrm{C}$ (day/night), the PPFD was $100 \mu \mathrm{mol} \mathrm{m} \mathrm{m}^{-2} \mathrm{~s}^{-1}$. The seedlings of two treatments with the same regular water and fertilizer management were exposed to relative humidity of $70 \%$ and 12 -h photoperiod (day/night). Each variety was repeated three times with 60 plants per repetition. Random sampling and index determination of functional leaves (3-4 leaves completely expanded from top to bottom) were carried out. 
Assessment of chilling injury index (CI): The chilling injury index was calculated according to Zhang et al. (2020a) after $20 \mathrm{~d}$ in darkness under $4^{\circ} \mathrm{C}$. The CI was calculated according to the equation: $\mathrm{CI}=(\Sigma n \times t) /(N \times T)$, where $n$ is the grade of one leaf, $t$ is the number of leaves of one grade, $N$ is the highest grade of one seedling, and $T$ is the total number of grades of one seedling.

Total RNA extraction, cDNA library construction, and sequencing: Total RNAs of CK and LL sample leaves of three biological replicates were isolated using a total RNA kit (TRIzol Reagent, Invitrogen, USA). RNA was assessed for its mass and concentration by $1 \%$ agarose gels electrophoresis and Nanodrop ND-1000 spectrophotometer (Thermo, USA), and RNA integrity was tested using an Agilent 2100 Bioanalyzer (Agilent Technologies, USA). Equal amounts of poly-A mRNA were enriched by Oligo magnetic beads and randomly broken into short NBE fragmentation buffer containing bivalent cation. Using the above mRNA as a template, first-strand cDNA synthesis was performed using random oligodeoxynucleotide hexamer primer and reverse transcriptase, and secondstrand cDNA was synthesized using DNA polymerase I and RNase H. cDNA fragments with $200 \mathrm{bp}$ after adding end-repair and nucleotide $\mathrm{A}$ addition were chosen by AMPure XP beads. The libraries were sequenced on an Illumina HiSeq 4000 platform (Illumina, USA) at Novogene company.

Quality control and differentially expressed genes (DEGs) screening: Total RNA of three biological replications from $\mathrm{X} 2$ and $\mathrm{H} 2$ samples exposed to $\mathrm{CK}$ and LL for $15 \mathrm{~d}$, marked as CK_X2_1, CK_X2_2, CK_X2_3, $\mathrm{LL} \_\mathrm{X} 2 \_1, \mathrm{LL} \_\mathrm{X} 2 \_2, \mathrm{LL} \_\mathrm{X} 2 \_3, \mathrm{CK} \_\mathrm{H} 2 \_1, \mathrm{CK} \_\mathrm{H} 2 \_2$, $\mathrm{CK}^{-} \mathrm{H} 2-3, \mathrm{LL}^{-} \mathrm{H} 2{ }^{-} 1, \mathrm{LL}^{-} \mathrm{H} \overline{2} 2$, and $\overline{\mathrm{L}} \overline{\mathrm{L}} \mathrm{H} 23$, were sequenced using Illumina $\bar{H} i \mathrm{Seq} 4000$ platform. The raw reads from mRNA-Seq were filtered by discarding reads containing adapters, low-quality reads with over $50 \%$ low $\mathrm{Q}$-value $\left(\mathrm{Q}_{\text {phred }} \leq 20\right)$, and reads with more than $10 \%$ unknown nucleotides (Chen et al. 2014a). High-quality clean reads in each of the 12 RNA-Seq data set were mapped against the $C$. annuum reference genome (https:// www.ncbi.nlm.nih.gov/) using TopHat software. The expression level of each gene was estimated according to the amounts of uniquely mapped reads by using the method of fragments per kilobase of transcript per million mapped reads (FPKM) in the program Cufflinks (version 1.0.3) (Trapnell et al. 2009). Differential expression analysis for CK and LL treatments of both $\mathrm{H} 2$ and $\mathrm{X} 2$ was performed, and then the differential analysis for DEGseq 2 (version 1.16.1) was used to identify the DEGs, with relative change threshold criteria of $\mid \log _{2}$ (fold change) $\mid>0$, false discovery rate $<0.005$, and $P_{\text {adj }}<0.05$.

Functional annotation: GO (http://www.geneontology. org/) and KEGG (https://www.genome.jp/kegg/) databases were used to annotate the DEG functions (Shi et al. 2011).

Validation of RNA-Seq data by qRT-PCR: Twenty transcripts were selected to verify RNA-Seq results. cDNA Synthesis Kit (TransStart Green, Beijing, China) was used for RNA reverse transcription and Primer 6.0 software was used for cross-exon primers designation. The qPCR SuperMix (TransStart Green, Beijing, China) and Bio-Rad CFX96 ${ }^{\mathrm{TM}}$ Real-Time System (Bio-Rad, CA, USA) were used to conduct qRT-PCR following the manufacturer's instructions. U6 was used to normalize the data, the relative expression of selected genes was calculated by the $2^{-\Delta \Delta C t}$ method. Information on the primers used in this study was listed in Table 1S (supplement).

Photosynthetic gas exchange, pigment content, chlorophyll fluorescence: The photosynthetic parameters of leaves, including transpiration rate $(E)$, stomatal conductance $\left(g_{\mathrm{s}}\right)$, net photosynthetic rate $\left(P_{\mathrm{N}}\right)$, and intracellular $\mathrm{CO}_{2}$ concentration $\left(C_{\mathrm{i}}\right)$, were measured using a portable photosynthesis system (CIRAS-2, PP-systems, UK) at 0 , $5,10,15$, and $20 \mathrm{~d}$ after treatment. The parameters for the instrument were set as follows: a PPFD of 1,000 $\mu \mathrm{mol}$

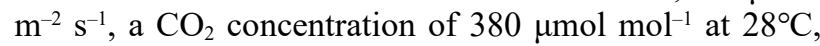
and relative humidity of $75 \%$.

The chlorophyll (Chl) $a, \mathrm{Chl} b, \mathrm{Chl}(a+b)$, and total carotenoid (Car) were determined after treatment for $15 \mathrm{~d}$ according to (Zhang et al. 2020a) using $80 \%$ acetone.

The Chl fluorescence was measured after treatment for 0, 5, 10, 15, and $20 \mathrm{~d}$ by using a fluorometer (Imaging PAM, Heinz Walz, Germany). Plants were dark-adapted for $30 \mathrm{~min}$ and the functional leaves were used for the measurement. The minimal fluorescence level $\left(\mathrm{F}_{0}\right)$ was measured after a modulated pulse of $280 \mu \mathrm{mol}$ (photon) $\mathrm{m}^{-2} \mathrm{~s}^{-1}$, maximal fluorescence $\left(\mathrm{F}_{\mathrm{m}}\right)$ was measured using a saturating actinic light. The maximum fluorescence yield $\left(\mathrm{F}_{\mathrm{m}}{ }^{\prime}\right)$, was recorded by kinetics curves of the saturation pulses applied after adapting plants to $320 \mathrm{~s}$ of illumination at $81 \mu \mathrm{mol}$ (photon) $\mathrm{m}^{-2} \mathrm{~s}^{-1}$ with a 20 -s interval (Genty et al. 1989, Kramer et al. 2004).

Carotenoid-related compounds: The extraction of ten carotenoid-related compounds, including Neo, Vio, Zea, Lut, $\beta$-Car, Lut-epoxide, antheraxanthin (Ant), lycopene (Lyc), phytoene (Phy), and $\alpha$-Car were performed after 15-d treatment according to Li et al. (2017). The extracts were passed through a $0.22-\mu \mathrm{m}$ millipore membrane and injected for high-performance liquid chromatography (HPLC) analysis. HPLC separation and quantification of carotenoids were performed on a Waters 2489 HPLC (Waters, Milford, MA, United States) equipped with a Waters 2487 dual $\lambda$ absorbance ultraviolet detector and a Welch Ultimate C30 column $(250 \times 4.6 \mathrm{~mm}$ i.d., $5 \mu \mathrm{m})$.

Statistical analyses: All data in the figures and tables are the means of three replicates, and error bars represent the standard deviation of three independent experiments. Duncan's multiple range test was carried out using SPSS 19.0 software for statistical analysis of the data. A $P<0.05$ was considered to be statistically significant. All figures were created in Origin ver. 8.5 and Adobe Photoshop CS 6. 


\section{Results}

The response of pepper seedlings to extremely low temperature and dark: After the application of lowtemperature and dark treatment $\left(5 / 5^{\circ} \mathrm{C}, 0 \mu \mathrm{mol} \mathrm{m} \mathrm{m}^{-2} \mathrm{~s}^{-1}\right)$ for $20 \mathrm{~d}, 7-8$ leaf pepper seedlings of $\mathrm{X} 2$ and $\mathrm{H} 2$ displayed serious visible morphological changes (Fig. 1). Most leaves of X2 remained green, and all stems of X2 lodged from the third to the fourth blade while most leaves of $\mathrm{H} 2$ lost their green color, scorched, and almost all stems lodged from the root base. These results suggest that X2 likely has stronger resistance to low-temperature and lowlight stress than that of $\mathrm{H} 2$, which is consistent with the statistical results; the CI of X2 (0.499) was significantly lower than that of $\mathrm{H} 2(0.809, P<0.05)$.

Transcriptome sequencing and assembly: Overviews of sequencing and assembly derived from 12 cDNA libraries were shown in Table 1. When adaptors and unknown or low-quality reads were filtered out, $82.24 \mathrm{~Gb}$ highquality clean reads $(274,147,815)$ were obtained from raw reads $(281,286,119)$ with an average of $6.85 \mathrm{~Gb}$ reads for each sample. The percentages of Q20 and Q30 base were not less than 95.48 and $93.23 \%$, respectively. The proportions of guanidine and cytosine nucleotides in the nucleotides' sequences were approximately $43 \%$ in 12 samples. Besides, 548,295,630 reads were unique mapped to the cultivated pepper reference genome of cv. Zunla-1 (C. annuum L.) (Qin et al. 2014), ranging from 83.12 to $88.12 \%$ per sample, which met the requirements for further information analysis (Table 1).

Comparative analyses of differentially expressed genes (DEGs): Pairwise comparisons between $\mathrm{CK}$ and LL obtained from the samples were identified to understand the differential expression genes under LL stress in pepper seedlings of both X2 and H2. A total of 8,392 DEGs (Table 2S, supplement) (4,018 upregulated and 4,374

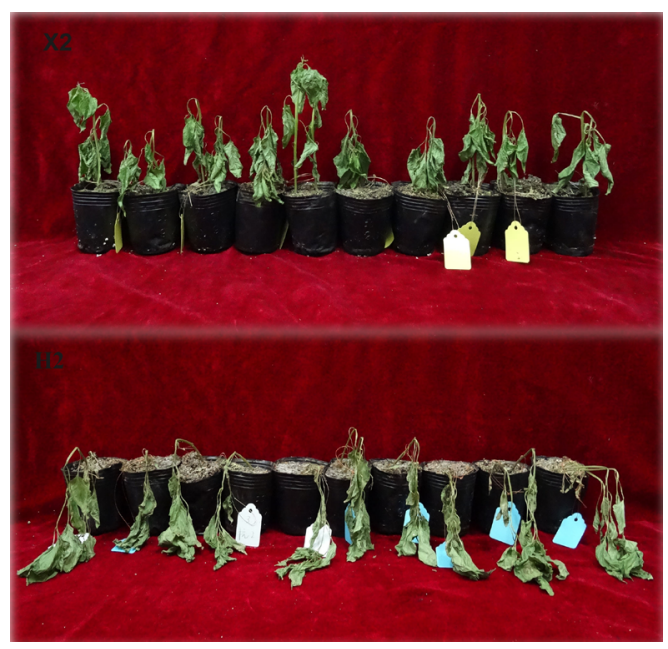

Fig. 1. Seedlings of X2 (Xiangtela No. 2) and H2 (Hangjiao No. 2) treated for $20 \mathrm{~d}$ under low temperature and dark $\left(5 / 5^{\circ} \mathrm{C}\right.$, day/night). The chilling injury (CI) was calculated. Data are the means of three independent replicates with ten seedlings.

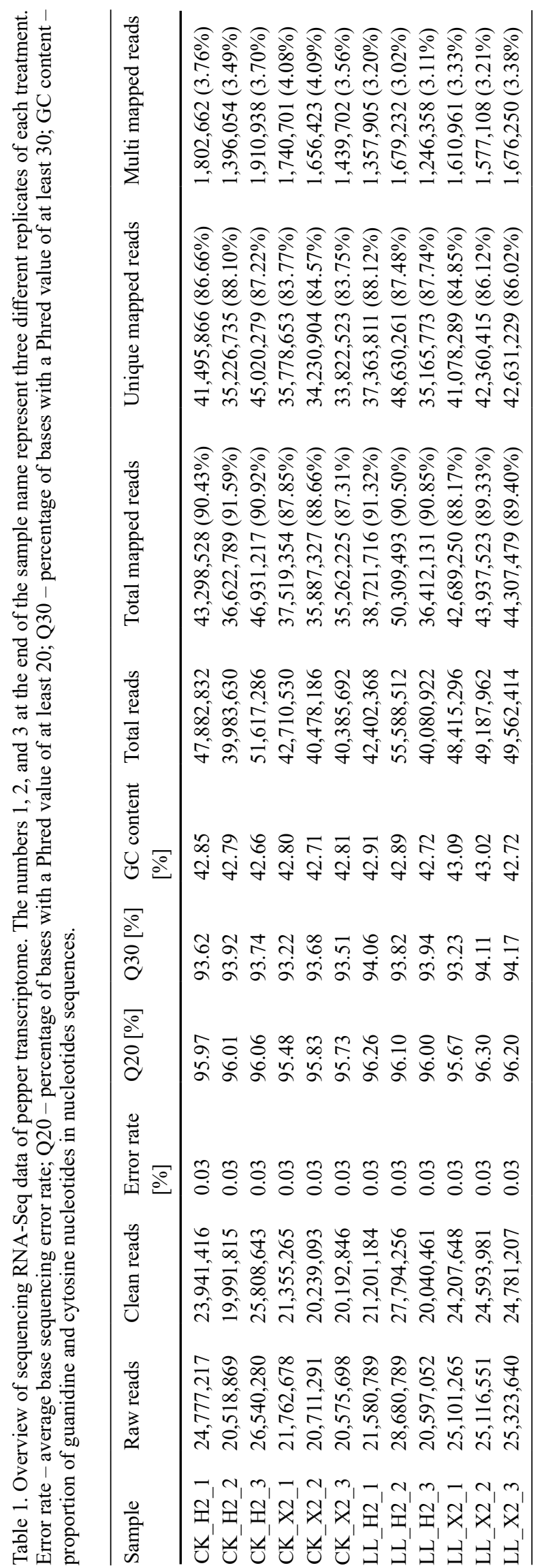


downregulated) from $\mathrm{H} 2$ groups (LL H2 vs. CK H2) and 8,028 DEGs (Table 3S, suplement) (4,284 upregulated and 3,744 downregulated) from the X2 group (LL_X2 vs. CK_X2) were identified (Fig. 2A), respectively. Furthermore, 4,933 common DEGs were found between the two groups but 3,459 and 3,095 DEGs were specific in the $\mathrm{H} 2$ and $\mathrm{X} 2$ groups, respectively (Fig. $2 B$ ).

Enriched GO analyses of DEGs: GO enrichment analysis was performed using the Blast $2 G O$ program to better understand the gene functions of DEGs. As shown in Fig. $3 A$, in $\mathrm{H} 2,1,128$ DEGs in the comparisons between the libraries of LL and CK were significantly annotated and classified into 27 subcategories of three main GO categories: biological process $(\mathrm{BP})$, cellular component $(\mathrm{CC})$, and molecular function $(\mathrm{MF})\left(P_{\mathrm{adj}}<0.05\right)$. Among these subcategories, 4, 9, and 14 were clustered in BP, $\mathrm{CC}$, and $\mathrm{MF}$, respectively. The four $\mathrm{GO}$ terms for $\mathrm{BP}$ were DNA-dependent DNA replication (GO: 0006261), photosynthesis (GO: 0015979), DNA replication initiation (GO: 0006270), and DNA replication (GO: 0006260). The top six GO terms for CC were thylakoid (GO: 0009579), thylakoid part (GO: 0044436), photosynthetic membrane (GO: 0034357), photosystem (GO: 0009521), photosystem II (GO: 0009523), and photosystem I (GO: 0009522). The top six GO terms for MF were microtubule binding (GO: 0008017), enzyme inhibitor activity (GO: 0004857), tubulin binding (GO: 0015631), enzyme regulator activity (GO: 0030234), microtubule motor activity (GO: 0003777) and heme binding (GO: 0020037).

In $\mathrm{X} 2$ (Fig. $3 B$ ), of the DEGs in the comparison between the libraries LL and CK, 2,745 were significantly assigned to three main GO categories with 39 subcategories $\left(P_{\mathrm{adj}}<0.05\right)$. Among the subcategories, 10, 20, and 9 were detected in BP with 1,055 genes enriched, CC with 1,110 enriched, and MF with 580 enriched, respectively. Besides, the top three GO terms for BP were peptide metabolic process (GO: 0006518), cellular amide metabolic process (GO: 0043603), and translation (GO: 0006412). The top ten GO terms for CC were ribosome (GO: 0005840), intracellular ribonucleoprotein complex (GO: 0030529), ribonucleoprotein complex (GO: 1990904), non-membrane-bounded organelle (GO: 0043228), intracellular non-membrane-bounded organelle (GO: 0043232), thylakoid (GO: 0009579), thylakoid part (GO: 0044436), cytoplasmic part (GO: 0044444), photosynthetic membrane (GO: 0034357), and photosystem (GO: 0009521). For the MF, the structural constituent of the ribosome (GO: 0003735) ranked first, followed by structural molecule activity (GO: 0005198) and transferase activity, transferring glycosyl groups (GO: 0016757).

Furthermore, the upregulated and downregulated DEGs of $\mathrm{H} 2$ between LL and CK were clustered into 46 subcategories of $\mathrm{BP}$ and MF and 17 of $\mathrm{BP}, \mathrm{CC}$, and MF. The top five significantly upregulated categories $\left(P_{\text {adj }}<0.01\right)$ included four from MF (microtubule-binding, GO: 0008017; tubulin binding, GO: 0015631; microtubule motor activity, GO: 0003777; and cytoskeletal protein

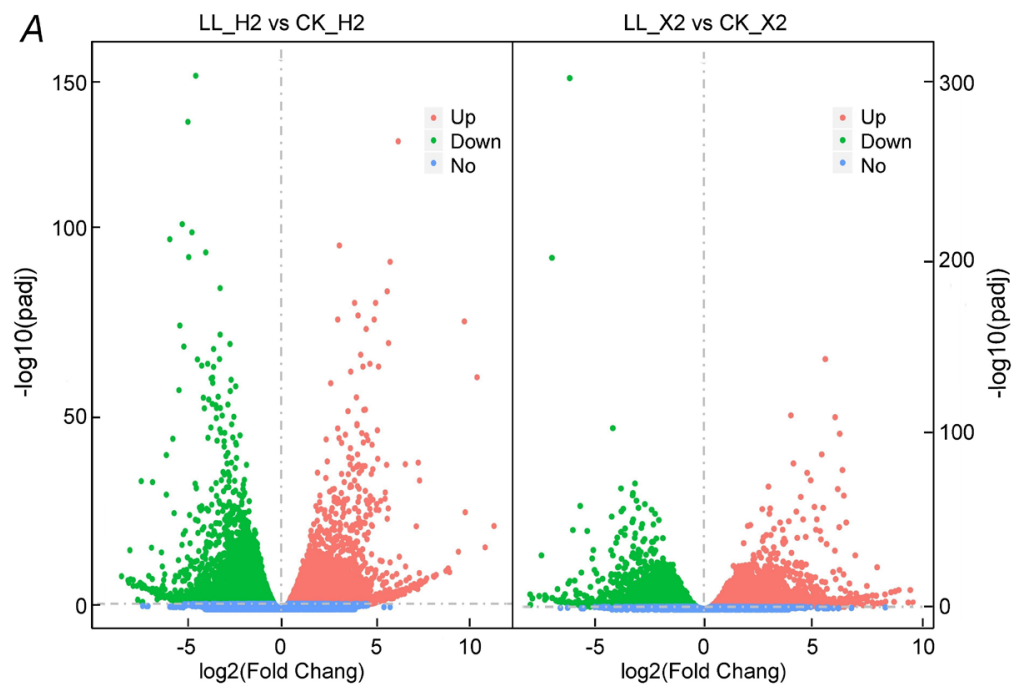

$B$

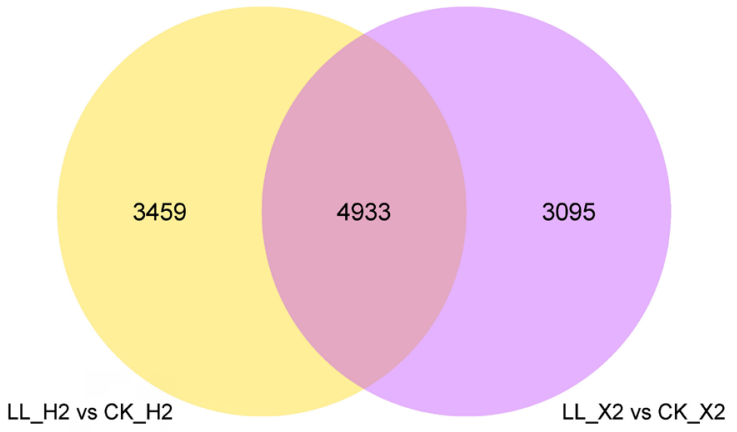

Fig. 2. Pepper genes identified to be differentially expressed (DEGs) between LL and control groups. (A) Volcanic plot of the differentially expressed genes. $(B)$ Venn diagram of DEGs between $\mathrm{H} 2$ group (LL H2 vs. CK H2) and $\mathrm{X} 2$ group (LL X2 vs. CK_X2). CK - normal temperature and light, $28 / 18^{\circ} \mathrm{C}$ and $300 \mu \mathrm{mol} \mathrm{m}^{-2} \mathrm{~s}^{-1}$; LL - low temperature combined with low light, $15 / 5^{\circ} \mathrm{C}$ and $100 \mu \mathrm{mol} \mathrm{m}^{-2} \mathrm{~s}^{-1}$. 


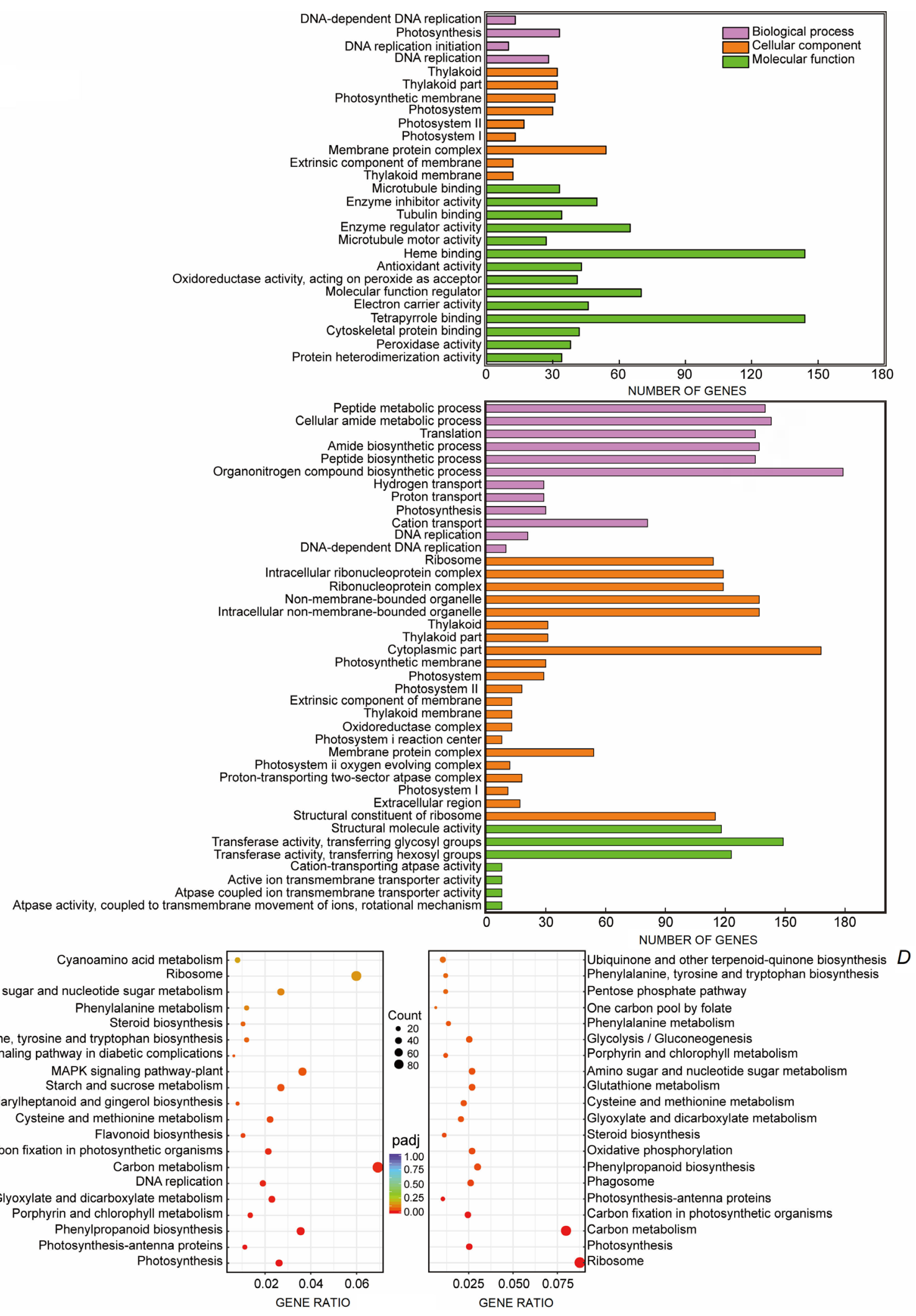

$B$

Fig. 3. GO and KEGG enrichment analyses of the differentially expressed genes between LL and control groups of pepper leaves. (A) GO analyses of the differentially expressed genes in H2 (Hangjiao No. 2) and (B) X2 (Xiangtela No. 2). (C) KEGG analyses of the differentially expressed genes in $\mathrm{H} 2$ (Hangjiao No. 2), and (D) X2 (Xiangtela No.2). CK - normal temperature and light, $28 / 18^{\circ} \mathrm{C}$ and $300 \mu \mathrm{mol} \mathrm{m} \mathrm{s}^{-1}$; LL - low temperature combined with low light, $15 / 5^{\circ} \mathrm{C}$, and $100 \mu \mathrm{mol} \mathrm{m}^{-2} \mathrm{~s}^{-1}$. 
binding, GO: 0008092), and one from BP (DNA replication, GO: 0006260). The top five significantly downregulated categories $\left(P_{\text {adj }}<0.01\right)$ included four from CC (thylakoid, GO: 0009579; thylakoid part, GO: 0044436; photosynthetic membrane, GO: 0034357; and photosystem, GO: 0009521) and one from BP (photosynthesis, GO: 0015979) (Table 4S, supplement). As for $\mathrm{X} 2$, the upregulated and downregulated DEGs between LL and CK were clustered into 84 subcategories of $\mathrm{BP}$ and MF and 17 of BP, CC, and MF. The top five significantly upregulated categories $\left(P_{\mathrm{adj}}<0.01\right)$ included two from MF (a structural constituent of ribosome, GO: 0003735, and structural molecule activity, GO: 0005198) and three from CC (ribosome, GO: 0005840; intracellular ribonucleoprotein complex, GO: 0030529; and ribonucleoprotein complex, GO: 1990904). The top five significantly downregulated categories $\left(P_{\text {adj }}<0.01\right)$ were the same as that of $\mathrm{H} 2$ (Table 5S, supplement).

Enriched KEGG pathway analyses of DEGs: The KEGG database was used to classify transcriptome DEGs into the pathway to elucidate the pathways of the DEGs involved in response to LL stress systematically. Compared with CK, 932 DEGs of $\mathrm{H} 2$ exposed to LL were enriched in 106 pathways, among which six pathways were significantly enriched $\left(P_{\text {adj }}<0.05\right)$ (Fig. 3C), including 35, 13, 17, 45, 30, and 23 DEGs enriched in photosynthesis (sly00195), photosynthesis-antenna proteins (sly00196), porphyrin and chlorophyll metabolism (sly00860), phenylpropanoid biosynthesis (sly00940), glyoxylate and dicarboxylate metabolism (sly00630), and DNA replication (sly03030),

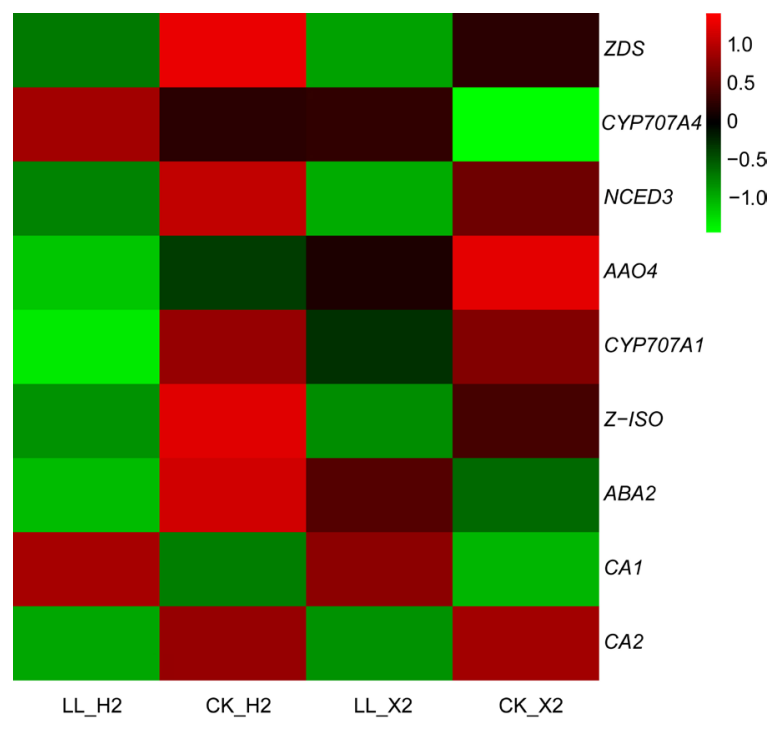

Fig. 4. Heat map of carotenoid differentially expressed genes of H2 (Hangjiao No. 2) and X2 (Xiangtela No. 2) under low light and temperature stress. ZDS - zeta-carotene desaturase; CA2 - beta-carotene hydroxylase 2; NCED3 - 9-cis-epoxycarotenoid dioxygenase; CA1 - beta-carotene hydroxylase 1; ABA2 - xanthoxin dehydrogenase-like; AAO4 - benzaldehyde dehydrogenase NAD(+)-like; CYP707A1 - abscisic acid 8'-hydroxylase 1-like; Z-ISO - 15-cis-zeta-carotene isomerase; CYP707A4-abscisic acid 8'-hydroxylase 4. respectively (Table $6 \mathrm{~S}$, supplement). In X2, 8,082 DEGs were enriched in 107 pathways, among which the five most significantly enriched were ribosome (sly03010), photosynthesis (sly00195), photosynthesis-antenna proteins (sly00196), carbon fixation in photosynthetic organism (sly00710), and carbon metabolism (sly01200) (Fig. 3D), in which 102, 33, 13, 31, and 85 DEGs were clustered, respectively (Table 7S, supplement).

Photosynthesis-related DEGs: Comparative transcriptome analysis between LL and $\mathrm{CK}$ in $\mathrm{H} 2$ and $\mathrm{X} 2$ revealed that many DEGs related to photosynthesis were involved in LL stress. The results of KEGG pathway annotation showed that the two pathways of photosynthesis (Table $8 \mathrm{~S}$, supplement) and photosynthesis antenna (Table 9S, supplement) were significantly enriched. Meanwhile, GO enrichment displayed the DEGs in the biological processes of photosynthesis and cellular component of photosystems, photosystem I, photosystem II, and photosystem II oxygen-evolving complex were significantly enriched. Furthermore, except for ferredoxin (FD3) and ferredoxin-NADP reductase, most of the DEGs involved in photosynthetic pathways, including encoding components of the photosystem, were all downregulated under LL in $\mathrm{H} 2$ and X2 (Table 8S).

Carotenoid metabolism-related DEGs: Carotenoids play predominant roles in photoprotection and light harvesting. Our results revealed that eight and five DEGs were related to carotenoid biosynthesis in $\mathrm{H} 2$ and X2, respectively (Fig. 4; Table 10S, supplement). After LL stress for $15 \mathrm{~d}$, four DEGs displayed similar trends in transcript levels in both varieties, three genes were downregulated, including zeta-carotene desaturase (ZDS), beta-carotene hydroxylase 2 (CA2), and 9-cis-epoxycarotenoid dioxygenase 3 (NCED3) but only beta-carotene hydroxylase 1 (CA1) was upregulated. Additionally, the $\log _{2}$ (fold change) of xanthoxin dehydrogenase-like (ABA2), benzaldehyde dehydrogenase $N A D(+)$-like (AAO4), abscisic acid 8'-hydroxylase 1-like (CYP707A1), and 15-cis-zetacarotene isomerase (Z-ISO) significantly changed in $\mathrm{H} 2$ under LL. The expression of abscisic acid 8 '-hydroxylase 4 (CYP707A4) exhibited the same trend under LL, and the $\log _{2}$ (fold change) in X2 was significantly higher than that in $\mathrm{H} 2$.

Validation of RNA-Seq data by qRT-PCR analysis: The expression levels of 20 DEGs under LL stress randomly selected from $\mathrm{H} 2$ and $\mathrm{X} 2$ were detected using qRT-PCR to verify the reliability and reproducibility of each differentially expressed transcript obtained by RNASeq. These candidate genes showed positive correlations (Fig. 1S, supplement), with $R^{2}=0.71$ in $\mathrm{H} 2$ and 0.72 in $\mathrm{X} 2$, between RNA-Seq $\log _{2}$ (fold change) and qRT-PCR $\log _{2}$ (fold change) in $\mathrm{H} 2$ and $\mathrm{X} 2$, which displayed a good consistency between RNA-Seq and qRT-PCR. These results confirmed the reliability of the data.

Photosynthetic gas exchange, photosynthetic pigment contents, and chlorophyll: The effects of LL on photo- 
synthetic gas-exchange parameters $\left(E, g_{\mathrm{s}}, P_{\mathrm{N}}\right.$, and $\left.C_{\mathrm{i}}\right)$ in $\mathrm{H} 2$ and $\mathrm{X} 2$ were evaluated after being treated for $0,5,10,15$, and $20 \mathrm{~d}$ (Fig. 5). Under LL, the $E$ (Fig. 5A), $g_{\mathrm{s}}$ (Fig. 5B), and $P_{\mathrm{N}}$ (Fig. $5 C$ ) of both varieties dramatically declined from 0 to $20 \mathrm{~d}$ after treatment. Significant differences in $E, g_{\mathrm{s}}$, and $P_{\mathrm{N}}$ were found between $0,5,10$, and $15 \mathrm{~d}$ but no significant difference was observed between 15 and $20 \mathrm{~d}$ after treatment in both varieties. Specifically, the $E, g_{\mathrm{s}}$, and $P_{\mathrm{N}}$ in $\mathrm{H} 2$ declined by $85.7,90.9$, and $94.9 \%$, whereas those in $\mathrm{X} 2$ decreased by $82.3,86.9$, and $71.3 \%$, respectively. By contrast, $C_{\mathrm{i}}$ (Fig. 5D) significantly increased by 86.0 and $65.7 \%$.

Fig. 6 shows changes in the concentrations of photosynthetic pigments, including $\mathrm{Chl} a$ (Fig. 6A), Chl $b$ (Fig. 6B), Chl $(a+b)$ (Fig. 6C), and Car (Fig. 6D), under $\mathrm{CK}$ and LL after $15 \mathrm{~d}$ of treatment in $\mathrm{H} 2$ and X2. These pigments were significantly lower in the LL-treated seedlings than that in the control seedlings. The concentrations of Chl $a$, Chl $b$, Chl $(a+b)$, and Car decreased by 54.0, $72.1,69.3$, and $43.3 \%$, respectively, in $\mathrm{H} 2$, and by 16.9 , $30.6,20.6$, and $7.7 \%$ in X2, respectively, showing a much smaller decline in $\mathrm{X} 2$ than that in $\mathrm{H} 2$.

The effects of LL on the Chl fluorescence parameters in $\mathrm{H} 2$ and X2 were evaluated after $0,5,10,15$, and $20 \mathrm{~d}$ of treatment. The $\mathrm{F}_{\mathrm{v}} / \mathrm{F}_{\mathrm{m}}$ of both varieties decreased with treatment time (Fig. 2S, supplement; Table 11S, supplement). $\mathrm{F}_{\mathrm{v}} / \mathrm{F}_{\mathrm{m}}$ decreased first on the leaf margin, the leaves exhibited damage on day 5 and severe damage on day 20. Although the $\mathrm{F}_{\mathrm{v}} / \mathrm{F}_{\mathrm{m}}$ of $\mathrm{H} 2$ obtained on day 20 was lower than that on day 15 , no significant differences were found after $15 \mathrm{~d}$. Besides, X2 displayed stronger LL tolerance than $\mathrm{H} 2$ and a slight decrease in $\mathrm{F}_{\mathrm{v}} / \mathrm{F}_{\mathrm{m}}$ at the blade edge after $10 \mathrm{~d}$. The veins exhibited no damage, whereas the leaves of $\mathrm{H} 2$ were damaged.

Carotenoid-related compounds: The concentrations of carotenoid-related compounds (Neo, Vio, Ant, Lut, Zea,
Lut-epoxide, Lyc, Phy, $\alpha$-Car, and $\beta$-Car) in the pepper leaves of $\mathrm{H} 2$ and X2 (Fig. 7; Fig. 3S, supplement) varied in response to LL. Compared with those of the CK seedlings, the Neo, Vio, Lut, Zea, Phy, and $\beta$-Car contents of the LL-treated seedlings significantly increased by 32.0 , $89.8,163.2,625.0,22.6$, and $81.2 \%$ in $\mathrm{H} 2$ and by 66.5 , $73.4,217.6,767.6,112.2$, and $111.0 \%$ in $\mathrm{X} 2$, respectively. Notably, the largest increase in both varieties under LL was found in Zea with 7.25-8.85-fold compared with CK. By contrast, Ant and Lut-epoxide significantly declined by 46.7 and $38.6 \%$ in $\mathrm{H} 2$, respectively, and by 37.4 and $80.7 \%$ in X2, respectively. Meanwhile, Lyc and $\alpha$-Car showed no significant difference in both varieties.

\section{Discussion}

Response to low-temperature or low-light stress alone via physiological and biochemical methods or gene expression detection has been studied previously (Chen et al. 2014b, Barrero-Gil et al. 2016, Li et al. 2016, Wang et al. 2018a, Mishra et al. 2019). However, few studies have focused on the underlying responsive mechanism when combining low-temperature and low-light stress, especially from the perspective of the transcriptome. To better understand the mechanism of LL stress response in pepper seedlings, this study focused on photosynthetic gas exchange, chlorophyll fluorescence parameters, photosynthetic pigment contents, carotenoid-related compound contents, and transcriptome sequences under $15 / 5^{\circ} \mathrm{C}$ for $15 \mathrm{~d}$. Our research showed that LL significantly altered physiological functions and biochemical contents. Series of transcription levels were changed as well as a large number of DEGs were also involved in diverse pathways such as photosynthesis, carotenoid metabolism, etc.

GO analysis is effectively used to identify the predominant biological processes associated with stress responses. In our research, DEGs of $\mathrm{H} 2$ and X2 pepper

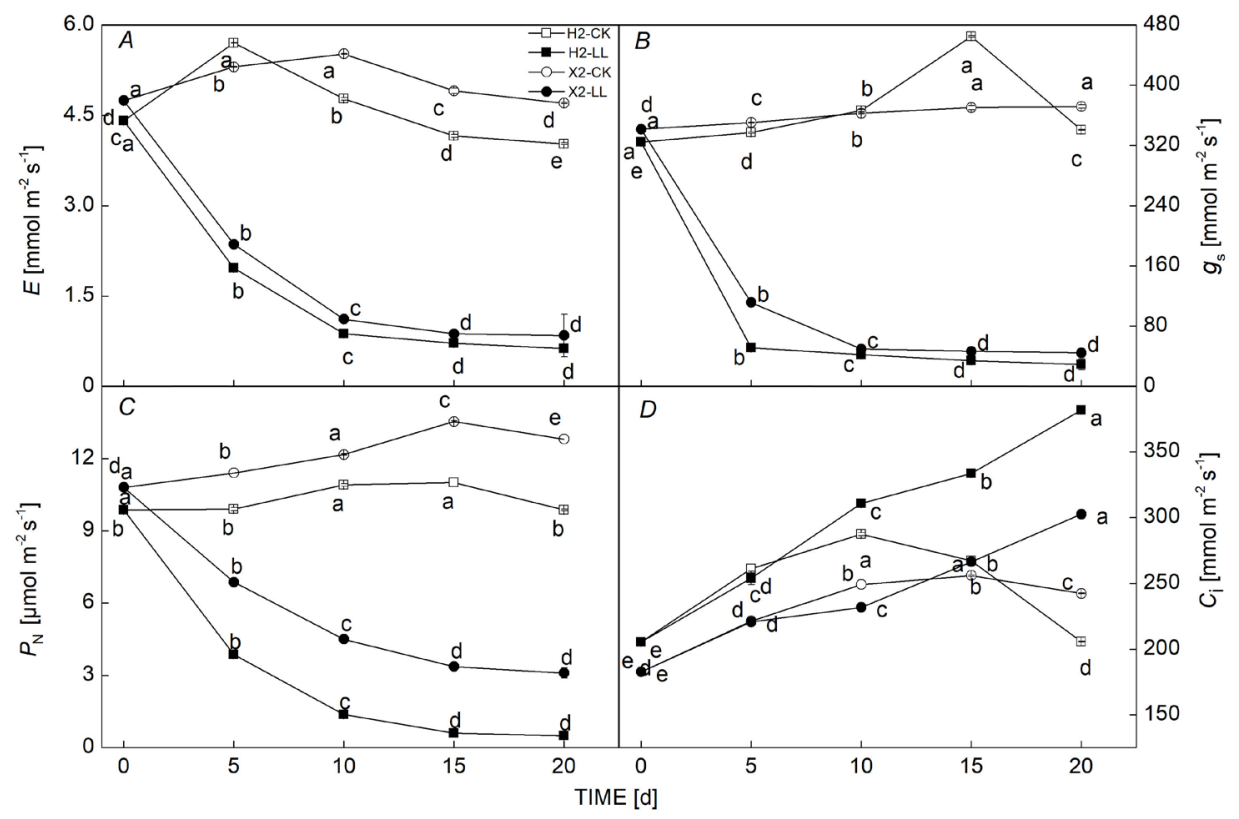

Fig. 5. Changes in photosynthetic gas exchange in the leaves of cultivars H2 (Hangjiao No. 2) and X2 (Xiangtela No. 2) under treatments for $0,5,10,15$, and 20 d. CK - normal temperature and light, $28 / 18^{\circ} \mathrm{C}$, and $300 \mu \mathrm{mol}$ $\mathrm{m}^{-2} \mathrm{~s}^{-1}$; LL - low temperature combined with low light, $15 / 5^{\circ} \mathrm{C}$, and $100 \mu \mathrm{mol} \mathrm{m}^{-2} \mathrm{~s}^{-1}$. (A) Transpiration $(E) ; \quad(B)$ stomatal conductance $\left(g_{\mathrm{s}}\right) ;(C)$ net photosynthetic rate $\left(P_{\mathrm{N}}\right)$; and $(D)$ intercellular $\mathrm{CO}_{2}$ concentration $\left(C_{\mathrm{i}}\right)$. Data in the graphs are means \pm SE from three independent experiments. Mean values with different letters in one line are significantly different from other treatment day $(P<0.05)$. 


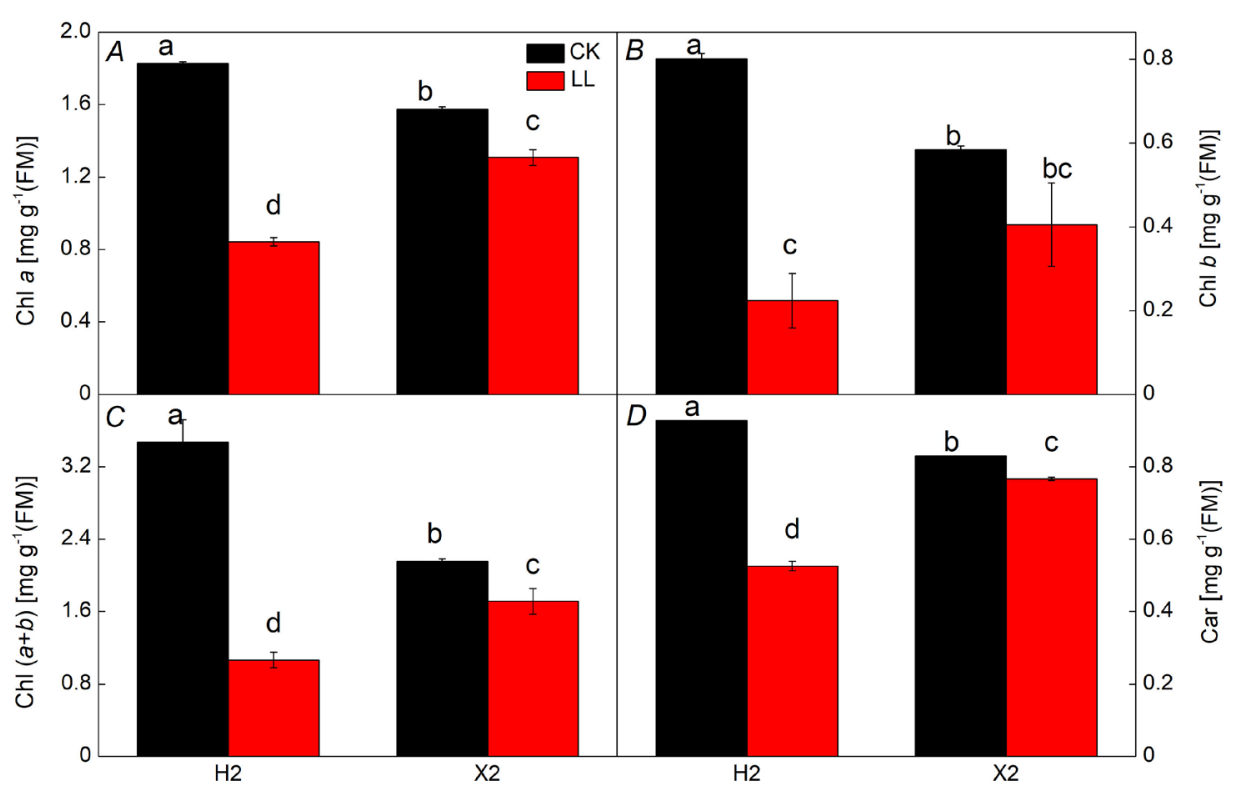

Fig. 6. Changes in photosynthetic pigment accumulation in the leaves of cultivars H2 (Hangjiao No. 2) and X2 (Xiangtela No. 2) under CK (normal temperature and light, $28 / 18^{\circ} \mathrm{C}$, and $300 \mu \mathrm{mol}$ $\mathrm{m}^{-2} \mathrm{~s}^{-1}$ ) and LL (low temperature combined with low light, $15 / 5^{\circ} \mathrm{C}$, and $100 \mu \mathrm{mol} \mathrm{m} \mathrm{m}^{-2} \mathrm{~s}^{-1}$ ) for $15 \mathrm{~d}$. (A) Chlorophyll a content (Chl $a) ;(B)$ chlorophyll $b$ content $(\mathrm{Chl} b) ;(C)$ total chlorophyll content [Chl $(a+b)]$; $(D)$ total carotenoid content (Car). Values are means \pm SE from three independent experiments. Values with different letters are significantly different from the other treatments $(P<0.05)$. seedlings under LL stress were enriched in 11 common GO terms in MF, such as photosynthesis, thylakoid part, photosynthetic membrane, photosystem, photosystem I, photosystem II, and so on (Fig. 3), indicating that DEGs under LL stress were involved in these pathways, which agrees with the previous study that two (photosynthesis and thylakoid) of 11 studied in our research also play a vital role in response to cold stress in indica rice (Pradhan et al. 2019). In the BP category, only three GO common terms were enriched in the two varieties in our present study. The DEGs were enriched in cellular amide metabolic process, peptide metabolic process, organonitrogen compound biosynthetic process, hydrogen transport, and proton transport in the tolerant variety X2 compared to susceptible variety $\mathrm{H} 2$; these differences may occur due to the better tolerance to LL stress in X2 than that of H2. In the CC category, the absence of related genes enriched significantly in the ribosome, intracellular ribonucleoprotein complex, ribonucleoprotein complex, non-membrane-bounded organelle, intracellular non-membrane-bounded organelle, cytoplasmic part, oxidoreductase complex, photosystem I reaction center, photosystem II oxygen-evolving complex, proton-transporting two-sector ATPase complex, and extracellular region in the variety of $\mathrm{H} 2$ might increase its susceptibility to LL stress.

In KEGG pathway analysis, genes involved in LL stress response were predicted to function in photosynthesis, photosynthesis-antenna proteins, carbon metabolism, phenylpropanoid biosynthesis, carbon fixation in photosynthetic organisms, porphyrin and chlorophyll metabolism, glyoxylate and dicarboxylate metabolism, cysteine and methionine metabolism (Fig. 3); a similar result was observed in Camellia oleifera 'Huaxin' leaf under cold stress, which also identified the genes involved in photosynthesis (Wu et al. 2020). Meanwhile, oxidative phosphorylation, steroid biosynthesis, glutathione metabolism, amino sugar and nucleotide sugar metabolism, glycolysis/gluconeogenesis, and phenylalanine metabo- lism were significantly enriched in $\mathrm{X} 2$ but not in $\mathrm{H} 2$, indicating that $\mathrm{X} 2$ is more tolerant to LL. This result conforms with the previous research findings that LL caused the changes in genes participating in respiration and metabolism of carbohydrates, phenylpropanoids, and antioxidants (Ou et al. 2015). Our results suggested that LL affected pepper photosynthesis capacity and photosynthetic pigments and might increase the energy of plant synthesis and metabolism. Besides, the synthesis and decomposition of organic matter might be enhanced to change osmotic adjustment substances, and antioxidant systems in plants could be stimulated. All those processes contributed to the improvement in the resistance of pepper.

Photosynthesis is a fundamental and intricate physiological process in plants involving photosynthetic pigments, photosystems, electron transport system, and $\mathrm{CO}_{2}$ reduction pathways, which are easily affected by abiotic stresses (Ashraf and Harris 2013, Abdelaal et al. 2020b, Alkahtani et al. 2020). Based on our results, LL decreased photosynthesis capacity $\left(E, P_{\mathrm{N}}, g_{\mathrm{s}}\right.$, and $\mathrm{F}_{\mathrm{v}} / \mathrm{F}_{\mathrm{m}}$ ) (Figs. 5, 2S) and numerous DEGs of both varieties significantly enriched in photosynthesis (sly00195), photosynthesis-antenna proteins (sly00196), and porphyrin and chlorophyll metabolism (sly00860). Therefore, photosynthesis is likely to be a predominant process in response to LL stress of pepper seedlings, which agrees with the findings in Magnolia wufengensis, Camellia oleifera 'Huaxin', and pea (Lucau-Danila et al. 2012, Deng et al. 2019, Wu et al. 2020). The light-harvesting oxygenic photosynthesis protein present in all eukaryotic organisms mainly includes the light-harvesting complex (Lhc) family members. Lhc are membrane intrinsic proteins: Lhca and Lhcb groups, which serve as photosystem I and photosystem II antenna, respectively, and noncovalently bind to Chl $a, \mathrm{Chl} b$, and carotenoids (lutein, neoxanthin, violaxanthin, and zeaxanthin) (Kirilovsky and Büchel 2019, Song et al. 2020). LL stress decreases photosynthesis (Zhang et al. 2020a) and causes photosynthetic organisms 


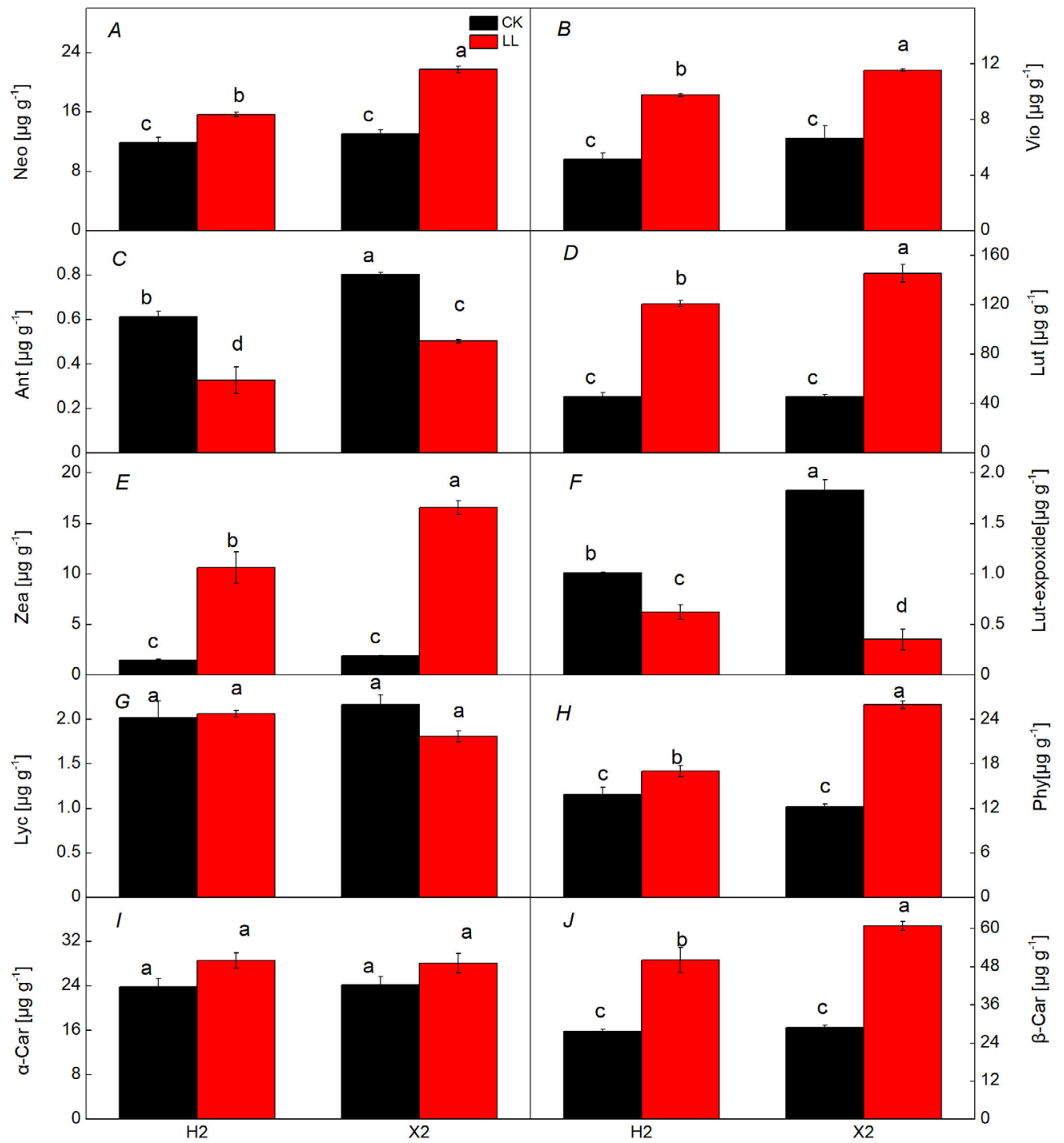

Fig. 7. Changes in the carotenoid compositions in the leaves of cultivars H2 (Hangiiao No. 2) and X2 (Xiangtela No. 2) after $15 \mathrm{~d}$ of treatments. $\mathrm{CK}$ - normal temperature and light, $28 / 18^{\circ} \mathrm{C}$, and $300 \mu \mathrm{mol} \mathrm{m}^{-2} \mathrm{~s}^{-1} ; \mathrm{LL}$ - low temperature combined with low light, $15 / 5^{\circ} \mathrm{C}$, and $100 \mu \mathrm{mol} \mathrm{m}^{-2} \mathrm{~s}^{-1}$. (A) Neoxanthin (Neo); (B) violaxanthin (Vio); (C) antheraxanthin (Ant); $(D)$ lutein (Lut); (E) zeaxanthin (Zea); $(F)$ lutein-epoxide (Lut-epoxide); $(G)$ lycopene (Lyc); $(H)$ phytoene (Phy); $(I) \alpha$-carotenoid $(\alpha$-Car); $(J) \beta$-carotenoid $(\beta$-Car). Each value (mean $\pm \mathrm{SD}$ ) represents the average of three independent replicates. Different letters denote statistically significant differences $(P<0.05)$ from the other treatments based on Duncan's multiple range test.

damage by excessive energy. Lhc family members are involved in the nonphotochemical quenching that accelerates heat dissipation depending on a $\mathrm{pH}$ gradient in the thylakoid membrane and xanthophyll de-epoxidation (Kirilovsky and Büchel 2019). Our study showed that photosynthetic pigments, such as $\mathrm{Chl} a, \mathrm{Chl} b$, and Chl $(a+b)$, significantly decreased after the treatment for $15 \mathrm{~d}$ under LL in both $\mathrm{H} 2$ and X2 (Fig. 6). Wu et al. (2020) also found a significant decrease in Chl, $P_{\mathrm{N}}$, and ETR as a result of photoinhibition and downregulation of Lhca and Lhcb under $6^{\circ} \mathrm{C}$ in two-year-old $C$. oleifera plants. Also, four genes in the photosynthesis pathway were related to photosystem complex structure in maize treated under $4^{\circ} \mathrm{C}$ for $24 \mathrm{~h}$, showing cold stress impaired the photosystem structure and resulted in low values of $\mathrm{F}_{\mathrm{v}} / \mathrm{F}_{\mathrm{m}}$ (Li et al.
2019), which is consistent with our study.

Carotenoids are a large group of naturally present pigments associated with vital plant physiological processes, photooxidative activities, and membrane stabilizers (Havaux 2014, Llorente 2016). Although previous studies have elucidated that carotenoids can alleviate the damage induced by stress, the regulatory mechanisms have received minimal attention. In the present study, significant differences could be obtained between LL and CK in both varieties concerning carotenoid concentration. The different treatments varied not only in the total carotenoid contents (Fig. 6D) but also in the qualitative distribution in zeaxanthin, lutein, and $\beta$-carotene (Figs. 7, 3S). Similarly, Zhang et al. (2020b) reported that Lut and $\beta$-Car increase in 'Qitouhuang' 
carrot under salt stress conditions, which might contribute to the upregulation of DcPSY2 (Zhang et al. 2020b). These results indicated Lut and $\beta$-Car might be involved in stress responses. Also, reports on tomato showed that $\beta$-carotene, lycopene, lutein, and violaxanthin contents significantly increased as a result of high expression levels of key genes in the carotenoid biosynthesis pathway, such as $P D S, Z D S$, $P S Y 1$, and $L Y C B$ under salt stress (Leiva-Ampuero et al. 2020). This is different from our results. Lycopene did not change significantly under LL stress, which may be due to the difference in stresses and plant species.

DEGs analyses revealed that LL triggered $Z D S, C A 2$, and NCED downregulation and $C A 1$ upregulation in $\mathrm{H} 2$ and X2 (Fig. 4), suggesting that LL induced changes in carotenoid accumulation. It is reported that overexpression of ZDS, PDS, and CRTISO of Lycium chinense in tobacco dramatically increased carotenoid contents and improved salt resistance and photosynthetic capacity by enhancing antioxidant enzyme activities ( $\mathrm{Li}$ et al. 2020). Thus, $Z D S$ might be involved in the desaturation process of carotenoids, its downregulation affects the homeostatic state and adapts to LL stress.

$C A 1$ and $C A 2$, two genes belonging to the $\beta$-carotene hydroxylase family ( $\beta$-carotenoid hydroxylase, $C H Y \beta)$, catalyze the synthesis of zeaxanthin from $\beta$-carotene. Genes $b 1$ and $b 2$ encode $C H Y \beta$ in Arabidopsis with highsimilarity sequences, while $b 1$ usually functions more than b2 (Moehs et al. 2001). Similarly, pepper has those two genes with $72 \%$ homology encoding $\beta$-hydroxylase (Bouvier et al. 1998). In the present study, the content of zeaxanthin increased significantly after $15 \mathrm{~d}$ under LL stress in both varieties, which may be caused by the promoted zeaxanthin synthesis by upregulating $C A 1$. Also, the fold of $C A 1$ upregulation in X2 was significantly higher than that in $\mathrm{H} 2$, which may explain the reason why the LL tolerance of X2 was significantly higher than that of $\mathrm{H} 2$. Many studies have shown that $C H Y \beta$ is associated with plant stress resistance, for instance, strong light, UV, high temperature, and biological stress resistance are significantly reduced in plants with $C H Y \beta$ deficiency (Davison et al. 2002). Meanwhile, Kang et al. (2017) revealed that downregulating the $C H Y \beta$ gene in transgenic sweet potato plants increased $\beta$-carotene content in the roots and leaves, thus causing improved tolerance to salt stress (Kang et al. 2017). Besides, the hydroxylation and epoxidation of lutein in plants are closely coordinated, the presence $\beta$-hydroxylase (chy2) is accompanied by downregulation of ZEP (zeaxanthin cyclooxygenase), resulting in a large accumulation of zeaxanthin that causes the orange meat phenotype of potato (Wolters et al. 2010). Recently, Wang et al. (2018b) have found that overexpressing tomato $\varepsilon$-hydroxylase genes can improve the drought resistance of transgenic tobacco plants and also induces SILUT1 expression under low-temperature stress (Wang et al. 2018b). Overall, it was inferred that $C A 1$ and $C A 2$ are related to LL stress.

Abscisic acid (ABA) is a vital downstream substance of carotenoid metabolism, 9-cis-epoxycarotenoid dioxygenase (NCED) and abscisic acid hydroxylase (CYP707A) are key enzymes in ABA synthesis. Yuan et al. (2018) have reported that plant development and stress resistance are regulated by $O S N C E D 5$, and overexpression of $O s N C E D 5$ increases $\mathrm{ABA}$ content enhancing the tolerance to salt stress, water stress, and darkness in rice (Yuan et al. 2018). Besides, CsNCED3 overexpression in tobacco enhances drought stress (Pedrosa et al. 2017), BnNCED3 overexpression in Arabidopsis significantly raises endogenous $\mathrm{ABA}$ contents and accelerates leaf senescence (Xu and Cai 2017), AtNCED3 and AtNCED5 overexpression improve water stress by controlling endogenous ABA contents (Pedrosa et al. 2017). Our results showed that $N C E D$ also played a significant role in LL stress, genes CYP707A1 (abscisic acid 8'-hydroxylase 1-like) and CYP707A4 (abscisic acid 8'-hydroxylase 4) encoding ABA-8'-hydroxylase expressed differently in the $\mathrm{LL}$ and $\mathrm{CK}$ groups of the two varieties, gene expression LOC107850059 inhibited by LL was compensated by upregulation of LOC107840293. ABA-8'-hydroxylase, a key enzyme in ABA metabolism, negatively regulates the content of endogenous ABA in plants. Tung et al. (2008) have shown that increased endogenous ABA content or exogenous spraying can inhibit the normal growth and development of plants (Tung et al. 2008). ABA can also regulate plant water use and improve drought resistance by controlling leaf stomatal closure (Takeuchi et al. 2016, Abdelaal et al. 2017). Consequently, it can be speculated that the LOC107840293 and LOC107850059 regulated ABA metabolism of pepper seedling under LL and then caused changes in LL tolerance further.

Conclusions: In the present study, upon RNA-Seq analysis of 12 libraries of LL-stress and control, a total of 8,392 and 8,028 differentially expressed genes were identified in varieties of $\mathrm{H} 2$ and $\mathrm{X} 2$; these genes are closely associated with biological processes, such as photosynthesis, photosynthesis antenna proteins, and carotenoids, etc. LL triggered $Z D S, C A 2$, and NCED downregulation and $C A 1$ upregulation in both varieties of $\mathrm{H} 2$ and $\mathrm{X} 2$, which may have participated in LL stress. Furthermore, LL significantly decreased photosynthesis, photosynthetic pigment contents, and $\mathrm{F}_{\mathrm{v}} / \mathrm{F}_{\mathrm{m}}$. Meanwhile, a significant increase in Neo, Vio, Lut, Zea, Phy, and $\beta$-Car was observed while a decrease in Ant and Lut-epoxide was found, revealing a role of carotenoids in response to LL stress. Our findings provide a theoretical basis for improved pepper breeding of LL-tolerant varieties.

\section{References}

Abdelaal K.A.A., Attia K.A., Alamery S.F. et al.: Exogenous application of proline and salicylic acid can mitigate the injurious impacts of drought stress on barley plants associated with physiological and histological characters. - Sustainability 12: 1736,2020 a.

Abdelaal K.A.A., El-Maghraby L.M., Elansary H. et al.: Treatment of sweet pepper with stress tolerance-inducing compounds alleviates salinity stress oxidative damage by mediating the physio-biochemical activities and antioxidant systems. - Agronomy 10: 26, 2020b.

Abdelaal K.A.A., Hafez Y.M., El-Afry M.M. et al.: Effect of some osmoregulators on photosynthesis, lipid peroxidation, 
antioxidative capacity, and productivity of barley (Hordeum vulgare L.) under water deficit stress. - Environ. Sci. Pollut. R. 25: 30199-30211, 2018.

Abdelaal K.A.A., Hafez Y.M., El Sabagh A., Saneoka H.: Ameliorative effects of abscisic acid and yeast on morphophysiological and yield characteristics of maize plant (Zea mays L.) under water deficit conditions. - Fresen. Environ. Bull. 26: 7372-7383, 2017.

Alkahtani M.D.F., Attia K.A., Hafez Y.M. et al.: Chlorophyll fluorescence parameters and antioxidant defense system can display salt tolerance of salt acclimated sweet pepper plants treated with chitosan and plant growth promoting rhizobacteria. - Agronomy 10: 1180, 2020.

An D., Yang J., Zhang P.: Transcriptome profiling of low temperature-treated cassava apical shoots showed dynamic responses of tropical plant to cold stress. - BMC Genomics 13: 64, 2012.

Ashraf M., Harris P.J.C.: Photosynthesis under stressful environments: An overview. - Photosynthetica 51: 163-190, 2013.

Barrero-Gil J., Huertas R., Rambla J.L. et al.: Tomato plants increase their tolerance to low temperature in a chilling acclimation process entailing comprehensive transcriptional and metabolic adjustments. - Plant Cell Environ. 39: 23032318, 2016.

Bouvier F., Keller Y., d'Harlingue A., Camara B.: Xanthophyll biosynthesis: molecular and functional characterization of carotenoid hydroxylases from pepper fruits (Capsicum annuит L.). - BBA-Lipid. Lipid Met. 1391: 320-328, 1998.

Campany C.E., Tjoelker M.G., von Caemmerer S., Duursma R.A.: Coupled response of stomatal and mesophyll conductance to light enhances photosynthesis of shade leaves under sunflecks. - Plant Cell Environ. 39: 2762-2773, 2016.

Cazzonelli C.I., Pogson B.J.: Source to sink: regulation of carotenoid biosynthesis in plants. - Trends Plant Sci. 15: 266274, 2010.

Chen D., Fu Y., Liu G., Liu H.: Low light intensity effects on the growth, photosynthetic characteristics, antioxidant capacity, yield and quality of wheat (Triticum aestivum L.) at different growth stages in BLSS. - Adv. Space Res. 53: 1557-1566, 2014 b.

Chen J., Kai H., Peng Q. et al.: RNA-Seq for gene identification and transcript profiling of three Stevia rebaudiana genotypes. - BMC Genomics 15: 571, 2014a.

Dall'Osto L., Cazzaniga S., Havaux M., Bassi R.: Enhanced photoprotection by protein-bound vs free xanthophyll pools: A comparative analysis of chlorophyll $b$ and xanthophyll biosynthesis mutants. - Mol. Plant 3: 576-593, 2010.

Daood H.G., Bencze G., Palotas G. et al.: HPLC analysis of carotenoids from tomatoes using cross-linked C18 column and MS detection. - J. Chromatogr. Sci. 52: 985-991, 2014.

Davison P.A., Hunter C.N., Horton P.: Overexpression of beta-carotene hydroxylase enhances stress tolerance in Arabidopsis. - Nature 418: 203-206, 2002.

Demmig-Adams B., Adams III W.W.: Photoprotection in an ecological context: the remarkable complexity of thermal energy dissipation. - New Phytol. 172: 11-21, 2006.

Deng S., Ma J., Zhang L. et al.: De novo transcriptome sequencing and gene expression profiling of Magnolia wufengensis in response to cold stress. - BMC Plant Biol. 19: 321, 2019.

Fratianni A., Mignogna R., Niro S., Panfili G.: Determination of lutein from fruit and vegetables through an alkaline hydrolysis extraction method and HPLC analysis. - J. Food Sci. 80: 2686-2691, 2015.

Genty B., Briantais J.M., Baker N.R.: The relationship between the quantum yield of photosynthetic electron transport and quenching of chlorophyll fluorescence. - BBA-Gen. Subjects 990: 87-92, 1989.

Hafez Y.M., Attia K.A., Alamery S. et al.: Beneficial effects of biochar and chitosan on antioxidative capacity, osmolytes accumulation, and anatomical characters of water-stressed barley plants. - Agronomy 10: 630, 2020.

Havaux M.: Carotenoid oxidation products as stress signals in plants. - Plant J. 79: 597-606, 2014.

Kang C., Zhai H., Xue L.Y. et al.: A lycopene $\beta$-cyclase gene, IbLCYB2, enhances carotenoid contents and abiotic stress tolerance in transgenic sweetpotato. - Plant Sci. 272: 243254, 2018.

Kang L., Ji C.Y., Kim S.H. et al.: Suppression of the $\beta$-carotene hydroxylase gene increases $\beta$-carotene content and tolerance to abiotic stress in transgenic sweetpotato plants. - Plant Physiol. Bioch. 117: 24-33, 2017.

Ke Q.B., Kang L., Kim H.S. et al.: Down-regulation of lycopene $\varepsilon$-cyclase expression in transgenic sweetpotato plants increases the carotenoid content and tolerance to abiotic stress. - Plant Sci. 281: 52-60, 2019.

Kim J.S., An C.G., Park J.S. et al.: Carotenoid profiling from 27 types of paprika (Capsicum annuum L.) with different colors, shapes, and cultivation methods. - Food Chem. 201: 64-71, 2016.

Kirilovsky D., Büchel C.: Evolution and function of lightharvesting antenna in oxygenic photosynthesis. - Adv. Bot. Res. 91: 247-293, 2019.

Kramer D.M., Johnson G., Kiirats O., Edwards G.E.: New fluorescence parameters for the determination of $\mathrm{Q}_{\mathrm{A}}$ redox state and excitation energy fluxes. - Photosynth. Res. 79: 209, 2004.

Legris M., Nieto C., Sellaro R. et al.: Perception and signalling of light and temperature cues in plants. - Plant J. 90: 683-697, 2017.

Leiva-Ampuero A., Agurto M., Matus J.T. et al.: Salinity impairs photosynthetic capacity and enhances carotenoid-related gene expression and biosynthesis in tomato (Solanum lycopersicum L. cv. Micro-Tom). - PeerJ 8: e9742, 2020.

Li C., Ji J., Wang G. et al.: Over-expression of $L c P D S, L c Z D S$, and LCCRTISO, genes from wolfberry for carotenoid biosynthesis, enhanced carotenoid accumulation, and salt tolerance in tobacco. - Front. Plant Sci. 11: 119, 2020.

Li J., Ping Y., Kang J.G. et al.: Transcriptome analysis of pepper (Capsicum annuum L.) revealed a role of 24-epibrassinolide in response to chilling. - Front. Plant Sci. 7: 1281, 2016.

Li J., Xie J.M., Yu J.H. et al.: Reversed-phase high-performance liquid chromatography for the quantification and optimization for extracting 10 kinds of carotenoids in pepper (Capsicum annuum L.) leaves. - J. Agr. Food Chem. 65: 8475-8488, 2017.

Li M., Sui N., Lin L. et al:: Transcriptomic profiling revealed genes involved in response to cold stress in maize. - Funct. Plant Biol. 46: 830-844, 2019.

Llorente B.: Regulation of carotenoid biosynthesis in photosynthetic organs. - In: Stange C. (ed.): Carotenoids in Nature. Subcellular Biochemistry. Pp. 141-160. Vol. 79. Springer, Cham 2016.

Lucau-Danila A., Toitot C., Goulas E. et al.: Transcriptome analysis in pea allows to distinguish chilling and acclimation mechanisms. - Plant Physiol. Bioch. 58: 236-244, 2012.

Mishra K.B., Mishra A., Kubásek J. et al.: Low temperature induced modulation of photosynthetic induction in nonacclimated and cold-acclimated Arabidopsis thaliana: chlorophyll $a$ fluorescence and gas-exchange measurements. Photosynth. Res. 139: 123-143, 2019.

Moehs C.P., Tian L., Osteryoung K.W., DellaPenna D.: Analysis 
of carotenoid biosynthetic gene expression during marigold petal development. - Plant Mol. Biol. 45: 281-293, 2001.

Ou L.J., Wei G., Zhang Z.Q. et al.: Effects of low temperature and low irradiance on the physiological characteristics and related gene expression of different pepper species. - Photosynthetica 53: 85-94, 2015.

Pedersen O., Colmer T.D., Borum J. et al.: Heat stress of two tropical seagrass species during low tides - impact on underwater net photosynthesis, dark respiration and diel in situ internal aeration. - New Phytol. 210: 1207-1218, 2016.

Pedrosa A.M., Cidade L.C., Martins C.P.S. et al.: Effect of overexpression of citrus 9-cis-epoxycarotenoid dioxygenase 3 (CsNCED3) on the physiological response to drought stress in transgenic tobacco. - Genet. Mol. Res. 16: gmr16019292, 2017.

Pradhan S.K., Pandit E., Nayak D.K. et al.: Genes, pathways and transcription factors involved in seedling stage chilling stress tolerance in indica rice through RNA-Seq analysis. - BMC Plant Biol. 19: 352, 2019.

Qin C., Yu C., Shen Y. et al.: Whole-genome sequencing of cultivated and wild peppers provides insights into Capsicum domestication and specialization. - P. Natl. Acad. Sci. USA 111: 5135-5140, 2014.

Rodriguez-Amaya D.B.: Structures and analysis of carotenoid molecules. - In: Stange C. (ed.): Carotenoids in Nature: Biosynthesis, Regulation and Function. Pp. 71-108. Springer, Cham 2016.

Sanghera G.S., Wani S.H., Hussain W., Singh N.B.: Engineering cold stress tolerance in crop plants. - Curr. Genomics 12: 3043, 2011.

Shi C.Y., Yang H., Wei C.L. et al:: Deep sequencing of the Camellia sinensis transcriptome revealed candidate genes for major metabolic pathways of tea-specific compounds. - BMC Genomics 12: 131, 2011.

Song H., Li Y., Xu X. et al.: Analysis of genes related to chlorophyll metabolism under elevated $\mathrm{CO}_{2}$ in cucumber (Cucumis sativus L.). - Sci. Hortic.-Amsterdam 261: 108988, 2020.

Takeuchi J., Okamoto M., Mega R. et al.: Abscinazole-E3M, a practical inhibitor of abscisic acid 8'-hydroxylase for improving drought tolerance. - Sci. Rep.-UK 6: 37060, 2016.

Thomashow M.F.: Plant cold acclimation: Freezing Tolerance Genes and Regulatory Mechanisms. - Annu. Rev. Plant Phys. 50: 571-599, 1999.

Trapnell C., Pachter L., Salzberg S.L.: TopHat: discovering splice junctions with RNA-Seq. - Bioinformatics 25: 1105$1111,2009$.

Truffault V., Gest N., Garchery C. et al.: Reduction of MDHAR activity in cherry tomato suppresses growth and yield and MDHAR activity is correlated with sugar levels under high light. - Plant Cell Environ. 39: 1279-1292, 2016.

Tricker P.J., Haefele S.M., Okamoto M.: The interaction of drought and nutrient stress in wheat: Opportunities and limitations. - In: Ahmad P. (ed.): Water Stress and Crop Plants: A Sustainable Approach. Pp. 695-710. John Wiley \& Sons, Chichester 2016.

Tung S.A., Smeeton R., White C.A. et al.: Over-expression of LeNCED1 in tomato (Solanum lycopersicum L.) with the rbcS3C promoter allows recovery of lines that accumulate very high levels of abscisic acid and exhibit severe phenotypes. Plant Cell Environ. 31: 968-981, 2008.

Wang K., Bai Z.Y., Liang Q.Y. et al.: Transcriptome analysis of chrysanthemum (Dendranthema grandiflorum) in response to low temperature stress. - BMC Genomics 19: 319, 2018 a.

Wang M., Zhang X., Liu J.H.: Deep sequencing-based characterization of transcriptome of trifoliate orange (Poncirus trifoliata (L.) Raf.) in response to cold stress. BMC Genomics 16: 555, 2015.

Wang S., Zhuang K., Zhang S. et al.: Overexpression of a tomato carotenoid $\varepsilon$-hydroxylase gene (SILUT1) improved the drought tolerance of transgenic tobacco. - J. Plant Physiol. 222: 103-112, 2018b.

Wolters A.M., Uitdewilligen J.G.A.M.L., Kloosterman B.A. et al:: Identification of alleles of carotenoid pathway genes important for zeaxanthin accumulation in potato tubers. Plant Mol. Biol. 73: 659-671, 2010.

Wu L., Li J., Li Z. et al.: Transcriptomic analyses of Camellia oleifera 'Huaxin' leaf reveal candidate genes related to longterm cold stress. - Int. J. Mol. Sci. 21: 846, 2020.

Xu P.P., Cai W.M.: Functional characterization of the BnNCED3 gene in Brassica napus. - Plant Sci. 256: 16-24, 2017.

Yuan H., Guo Y., Liu Y. et al.: 9-cis-epoxycarotenoid dioxygenase 3 regulates plant growth and enhances multi-abiotic stress tolerance in rice. - Front. Plant Sci. 9: 162, 2018.

Zhang J.F., Li J., Xie J.M. et al.: Changes in photosynthesis and carotenoid composition of pepper (Capsicum annuum L.) in response to low-light stress and low temperature combined with low-light stress. - Photosynthetica 58: 125-136, 2020a.

Zhang R.R., Wang Y.H., Li T. et al.: Effects of simulated drought stress on carotenoid contents and expression of related genes in carrot taproots. - Protoplasma, 2020b. doi: 10.1007/ s00709-020-01570-5.

Zhao J., Hartmann H., Trumbore S. et al.: High temperature causes negative whole-plant carbon balance under mild drought. - New Phytol. 200: 330-339, 2013.

Zhuang K., Kong F., Zhang S. et al.: Whirly1 enhances tolerance to chilling stress in tomato via protection of photosystem II and regulation of starch degradation. - New Phytol. 221: 1998-2012, 2019.

(C) The authors. This is an open access article distributed under the terms of the Creative Commons BY-NC-ND Licence. 